\title{
SOLID LIPID NANOPARTICLES - EFFECT OF LIPID MATRIX AND SURFACTANT ON THEIR PHYSICAL CHARACTERISTICS
}

\author{
Mamdouh M. Ghorab ${ }^{1}$, Heba M. Abdel-Salam² and Mohamed M. Abdel-Moaty ${ }^{2}$ \\ ${ }^{1}$ Department of Pharmaceutics, College of Pharmacy, Suez Canal University, Ismailia, Egypt \\ ${ }^{2}$ Research Projects Department, MUP Co., Abu-Sultan, Ismailia, Egypt
}

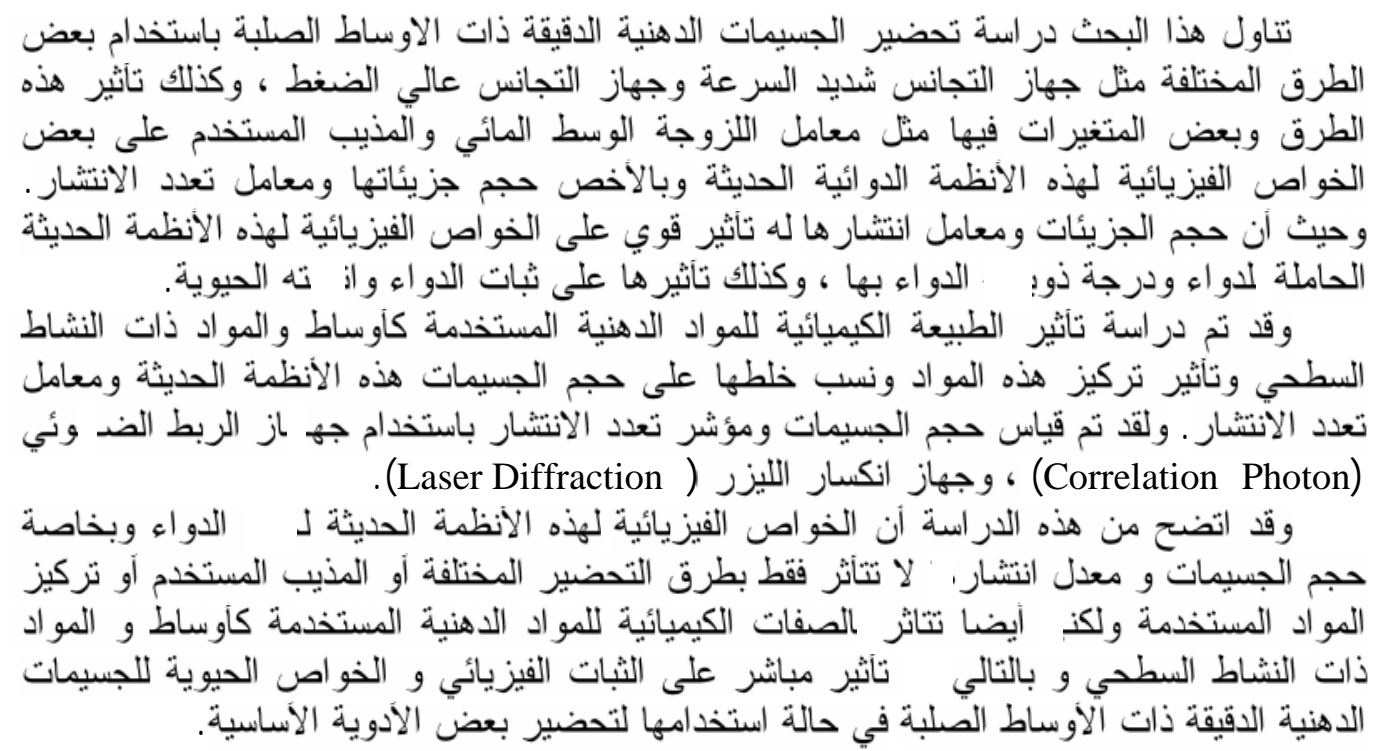

Solid lipid nanoparticles (SLN) have gained increasing attention as a colloidal drug carrier system, particularly for lipophilic drugs, because SLN combine the advantages of polymeric nanoparticles, fat emulsions, and liposomes but avoiding their disadvantages. Solid lipid nanoparticles are composed of high melting point lipid as a solid core coated by surfactants. The solid core allows the prolonged and controlled release of drugs and may protect incorporated drugs against chemical degradation. Lipid and surfactant nature are important in drug loading capacity, also affect size distribution and physical stability. In this study SLN were prepared by using different ratios of lipid/surfactant. The lipid nanopellets were produced by using the high speed homogenizer and by high pressure homogenizer. The particle size measurements were carried out using the photoncorrelation spectroscopy and laser diffraction. The results showed that the diameter of SLN were influenced by lipid matrix and surfactant nature as well as their concentrations. Particle size diameter was decreased as a function of surfactant concentration till it reach a certain limit after which it has no effect. Methods of preparation showed a potential effect on the presence of large particles and on the polydispersity index. In conclusion, physical characteristics of SLN are not only influenced by variation in process parameters but also by chemical nature of the used surfactant and lipid matrix that have a potential influence on the particle size distribution of SLN.

\section{INTRODUCTION}

Recently, solid lipid nanoparticles (SLN) have gained increasing attention as a colloidal drug carrier system, particularly for lipophilic drugs. ${ }^{1}$ Nanoparticles based on solid lipids have been proposed as a promising alternative colloidal drug delivery system as they combine the advantages of polymeric nanoparticles, fat emulsions, and liposomes but avoiding their disadvantages. A clear advantage of SLN over polymeric nanoparticles is the fact that lipid matrix is made from physiological lipids, which decrease the danger of acute and chronic 
toxicity. ${ }^{2}$ Solid lipid nanoparticles are composed of high melting point lipid as a solid core coated by surfactants. The solid core allows the prolonged and controlled release of drugs and may protect incorporated drugs against chemical degradation. Lipid and surfactant chemical natures are important in drug loading capacity, also affect size distribution and physical stability, ${ }^{1}$ that is to say the difference in fatty acids chain length and degree of glycerides substitution of the core lipids influence the overall lipid hydrophobicity which in turn may influence particle size distribution as well as SLN physical stability.

The objective of this study was to describe the effect of lipid matrix and surfactant nature, ratio, concentrations and method of preparation on the physical characteristics of the SLN (especially particle size distribution and polydispersity index).

\section{EXPERIMENTAL}

\section{Materials}

Compritol 888ATO (Glyceryl behenate) and Precirol ATO5 (Glyceryl distearate) Gattefosse, (France). Glyceryl monostearate (Quimasso, France). Stearic acid, (New port, Ireland). Tween 80 (Polysorpate 80) and Tween 20 (Polysorpate 20), ICI Americas (Wilmington, DE, USA). Ethanol, acetone and isopropanol and all other chemicals were of reagent grade and used without further purification.

High speed homogenizer (Ultra-Turrax T25, IKA, Germany) and high pressure homogenizer (Emulsiflex, C-5, Avestin, Canada). Particle size measurements were carried out using the photoncorrelation spectroscopy (Zetasizer 1000HSA, Malvern, UK) and laser diffraction (Shimadzu, SALD2001, Japan).

\section{Methods \\ Preparation of solid lipid nanoparticles}

Solid lipid nanoparticles were prepared by several techniques using various ratios of selected lipids and surfactants. Firstly, the lipid was melted $\left(60-70^{\circ}\right)$ and dispersed in hot aqueous solution with different surfactant concentrations $(0.5,1$ and $5 \% \mathrm{w} / \mathrm{v})$ at the same temperature, by high-speed stirring, using an
Ultra-Turrax homogenizer at $12,000 \mathrm{rpm}$ for 10 minutes, with 30 seconds intervals every two minutes. The obtained pre-emulsions were cooled to room temperature $\left(25^{\circ}\right)$ and their particle size was measured. ${ }^{3}$ For the hot melt technique the SLNs were prepared as before then the hot pre-emulsion furtherly passed through high-pressure homogenizer at temperature of $60-70^{\circ}$ and pressure of 500 bar for three cycles. ${ }^{3}$ Also, SLNs were prepared by a modified solvent injection technique, in which, the lipids were dissolved in a watermiscible solvent and then rapidly injected into a stirred (approx. 12,000 rpm) hot aqueous phase with surfactant. ${ }^{2}$ The resulting dispersion was then cooled and particle size was measured.

\section{Particle size measurements}

The particle size of the produced lipid nanoparticles was measured by using the photoncorrelation spectroscopy (PCS) using a Zetasizer 1000HSA, and laser diffraction. The measurements were investigated at an angle of $90^{\circ}$. Each sample was diluted with filtered bidistilled water $(1 \mathrm{~g}$ in $50 \mathrm{ml})$ until appropriate concentration of particles was achieved to avoid multiscattering events and measured with a sample time of $0.5 \mathrm{~ms}$ for $5 \mathrm{~min}$ in serial mode. ${ }^{4}$ Each measurement was performed in triplicate and the particle average diameter and polydispersity index (PI) were determined.

\section{Viscosity measurement}

The viscosity of the aqueous surfactant phase $(0.5 \% \mathrm{w} / \mathrm{v})$ was varied by the addition of 10,30 and $50 \%(\mathrm{w} / \mathrm{v})$ glycerol. The viscosity of the aqueous phase was determined using Brookfield viscometer, at room temperature.

\section{RESULTS AND DISCUSSION}

\section{Effect of preparation method}

To compare the efficiency of the dispersion methods, melted glyceryl monostearate (GMS) was dispersed in $0.5 \%$ Tween $80(\% \mathrm{w} / \mathrm{w})$ by means of (a) a stirrer (Ultra-Turrax, UT), (b) solvent injection and Ultra-Turrax homogenizer, and (c) by highpressure homogenization. The volume distribution of the lipid particles produced by UT treatment showed a maximum at approximately $1.4 \mu \mathrm{m}$ (Fig.1). Preparation by 
solvent injection and UT treatment had no significant effect on particle size distribution than that of the UT treatment alone, with maximum at approximately $1.3 \mu \mathrm{m}$. However, preparation of SLN by high-pressure homogenization proved to be the most effective in minimizing the number of large particles. The area under the distribution curve shows that no particles $>1 \mathrm{um}$. The results obtained are consistent with those obtained by Swharz et $a .^{3}$

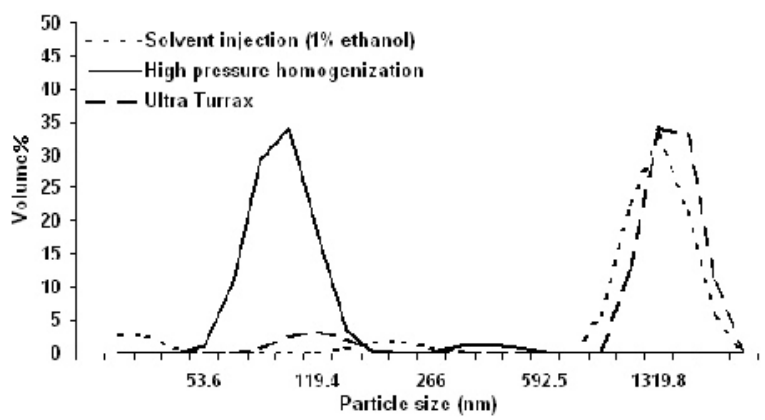

Fig 1: Effect of preparation technique on particles of $5 \%$ glyceryl monostearate lipid dispersion stabilized with $0.5 \%$ Tween 80 .

\section{Effect of solvent type used in solvent injection technique}

The influence of water miscible solvent (ethanol, isopropanol or acetone) on dispersity of lipid particles was studied, where a hot solution of GMS in a water-miscible solvent is rapidly injected into an aqueous phase at the same temperature containing $0.5 \% \quad(\mathrm{w} / \mathrm{w})$ Tween $80 .^{5}$ The data for all three studied solvents showed no significant difference in mean particle size (Z-average) and polydispersity index (PI) (Fig. 2). For the preparation conditions, isopropanol seems to be the best solvent in term of particle size $(367 \mathrm{~nm}$ $\pm 24.50)$ and PI (0.63 \pm 0.038$)$. However, ethanol

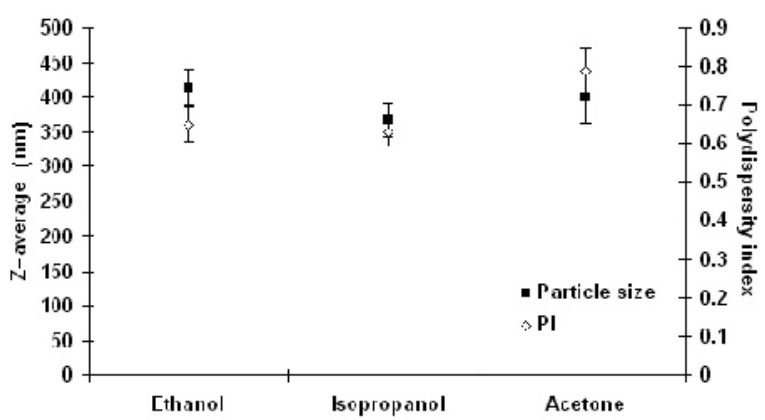

Fig 2: Particle size and polydispersity index (PI) versus type of solvent used $(n=3)$. and acetone are also suitable, were SLN produced had slight larger diameter $414 \mathrm{~nm}$ \pm 26.25 and $400 \mathrm{~nm} \pm 37.44$ and PI $0.65 \pm 0.045$ and $0.79 \pm 0.056$, respectively. This observation may be due to the degree of solubility of the used lipid (glyceryl monostearate) in the examined solvents is approximately the same and therefore equal rate of precipitation of the lipid in the aqueous phase.

\section{Effect of viscosity of the dispersion medium}

The viscosity of the dispersion medium influences the SLN particle size. ${ }^{5}$ Figure 3 illustrated that by increasing the viscosity of the aqueous phase by addition of different concentrations of glycerol, leads to a marked decrease of SLN particle size and PI. This result is not in agreement with the previous finding by Schubert et al., ${ }^{5}$ however using different lipid. This result suggests that increasing viscosity of the outer phase increase the impact rate between the lipid and the external phase, leading to decrease in particle size. However, further work in this area is required using different lipids and different viscosity imparting agents.

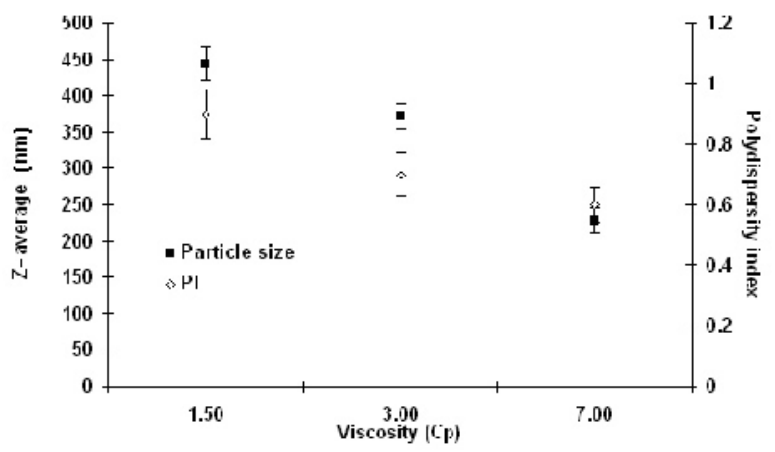

Fig 3: Particle size and polydispersity index (PI) versus viscosity of dispersion medium $(n=3)$.

\section{Effect of surfactant type and concentration}

To establish the effect of surfactant type on lipid dispersion, selected systems were used (5\% Glyceryl monostearate with $1 \%$ Tween 20 and Tween 80) and prepared by using UT treatment. Figure 4 showed a maximum distribution volume at approximately $1.3 \mu \mathrm{m}$ of the lipid particles and small fraction of smaller particles $<1 \mu \mathrm{m}$ was produced by using Tween 20 in the dispersion medium. However, addition of Tween 80 shifted the maximum of the particle distribution to smaller values 
$(<0.167 \mu \mathrm{m})$ with small fraction of particles $>1$ $\mu \mathrm{m}$. A possible explanation for this difference in particle size distribution may be the effect of hydrocarbon chain length difference of the used surfactants that affect on the aggregation number of non-ionic surfactant. ${ }^{6}$ Arnarson and Elworthy $^{7}$ have previously shown that increasing the hydrocarbon chain length in nonionic surfactants from $\mathrm{C} 16$ to $\mathrm{C} 22$ gave bigger micelles, but with low solubilizing capacity of lipophilic drugs. This decrease in solubilizing capacity, may be the reason for the formation of fine emulsion droplets in case of Tween 80 $(\mathrm{HLB}=15)$ that contain $\mathrm{C} 18$ (monooleate) fatty acid chain, and larger droplets in case of Tween $20(\mathrm{HLB}=16.7)$ that contain $\mathrm{C} 12$ (monolaurate) fatty acid chain. Also, the large difference in behavior brought about by the presence of double bond in the hydrocarbon side chain of Tween 80 than that of saturated one in Tween 20.

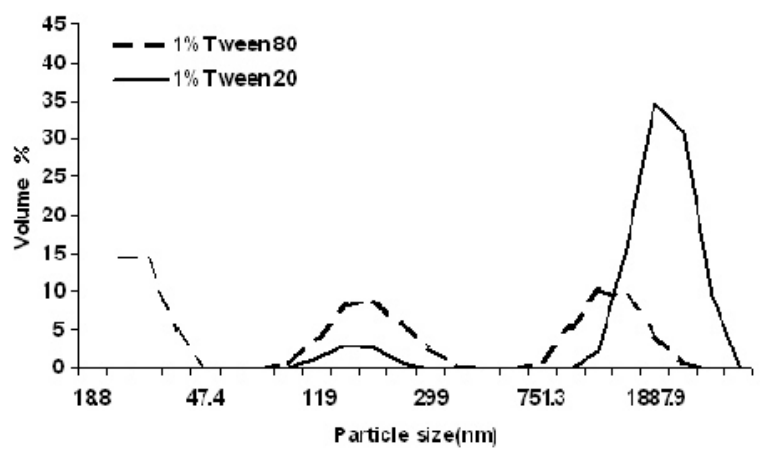

Fig 4: Solid lipid nanoparticles particle size distribution as a function of surfactant type.

The influence of emulsifier concentration on particle size is illustrated in Figure 5. It is clear that further addition of surfactant (Tween 80) lead to a further decrease of particle size till reach a certain limit after which it had no effect, though the particle size distribution remain more or less unchanged. The increase in the surface-active properties of surfactants by increasing surfactant amount in colloidal dispersions may be contribute to the reduction of mean particle size. ${ }^{8}$

Thus with regard to particle size the emulsifier type and concentration seems to be an important process parameters.

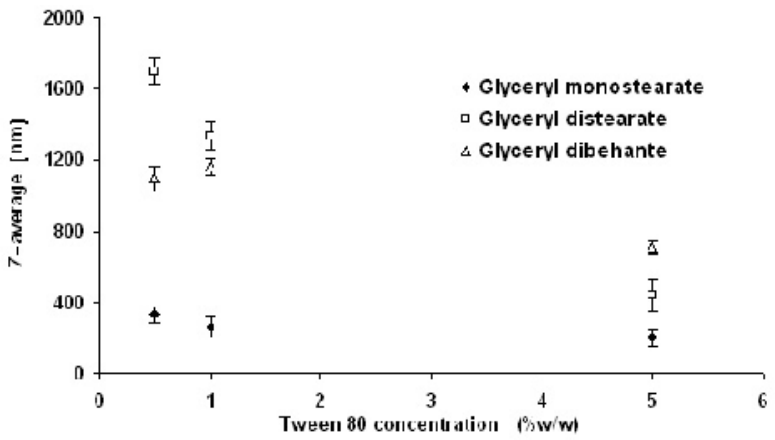

Fig 5: Effect of surfactant concentration on particle size for different lipid matrix.

\section{Effect of structural variations of lipid matrix}

Table 1 showed that the variation in particle size with surfactant concentrations depend upon the nature and the amount of lipid incorporated. For example, SLN dispersions prepared with stearic acid (most hydrophobic), exhibited first a decrease followed by marked increase then decrease in the average particle size (Z-average) upon increasing lipid content in presence of 5\% Tween 80, and no SLN was formed with high lipid concentration $(>5 \%)$ in presence of low surfactant concentrations (1\% Tween 80). However, with other lipids, namely the less lipophilic (more polar) glyceryl monostearate and glyceryl distearate this is not followed especially with low surfactant concentration $(1 \%)$, where a slight increase in particle size was seen as a function of lipid concentration, but the phenomenon of decrease in particle size followed by increase then decrease was appeared again with high surfactant concentration.

It is also evident from the obtained data that SLN formed with stearic acid had larger particle size than that of glyceryl distearate and SLN formed with glyceryl monostearate has the smallest particle size. This difference in lipophilicity of the used lipids not only affect average particle size but also PI, as the more lipophilic lipid (stearic acid and glyceryl distearate) produced SLN with PI >1; however, the less lipophilic glyceryl monostearate had PI $<1$. This indicated that the homogeneity of particle size population affected significantly with the nature of the lipid core as well as its concentration. 
Table 1: Mean particle size (nm), polydispersity index (PI) of dispersions containing different lipids percentage.

\begin{tabular}{|c|c|c|c|c|}
\hline \multirow{3}{*}{$\begin{array}{l}\text { Lipid Concen. } \\
\qquad(\% \mathrm{w} / \mathrm{w})\end{array}$} & \multicolumn{4}{|c|}{$\begin{array}{c}\text { Tween } 80 \text { at various concentrations } \\
(\% \mathrm{w} / \mathrm{w}) \text { : }\end{array}$} \\
\hline & \multicolumn{2}{|c|}{1} & \multicolumn{2}{|c|}{5} \\
\hline & Z-average * & PI & Z-average* & PI \\
\hline 1. Stearic acid & & & & \\
\hline 5 & $1663 \pm 98$ & $>1$ & $737 \pm 22$ & $>1$ \\
\hline 7.5 & -- & -- & $2957 \pm 143$ & $>1$ \\
\hline 10 & -- & -- & $731 \pm 55$ & $>1$ \\
\hline 2. Glyceryl & & & & \\
\hline monostearate & $264 \pm 14$ & 0.61 & $200 \pm 18$ & 0.58 \\
\hline 5 & $272 \pm 11$ & 0.40 & $359 \pm 9$ & 0.74 \\
\hline 7.5 & $358 \pm 5$ & 0.57 & $292 \pm 10$ & 0.66 \\
\hline 10 & & & & \\
\hline 3. Glyceryl distearate & $1337 \pm 73$ & $>1$ & $438 \pm 32$ & $>1$ \\
\hline 5 & $1417 \pm 68$ & $>1$ & $1627 \pm 90$ & $>1$ \\
\hline 7.5 & $1438 \pm 87$ & $>1$ & $965 \pm 82$ & $>1$ \\
\hline 10 & & & & \\
\hline 4.Glyceryl dibehnate & $1161 \pm 56$ & $>1$ & $711 \pm 33$ & $>1$ \\
\hline 5 & $2968 \pm 104$ & $>1$ & $1381 \pm 98$ & $>1$ \\
\hline 7.5 & $1610 \pm 43$ & $>1$ & $1076 \pm 15$ & $>1$ \\
\hline 10 & & & & \\
\hline
\end{tabular}

$(--=$ no SLN are formed $)$

$*( \pm$ S.D., $n=3)$

In conclusion, it has been demonstrated that particle size distribution of SLN not only affected and controlled by variation of process parameters such as methods of preparation, solvents used, viscosity of dispersion medium, surfactant type and concentration, but also with lipid matrix structure. The difference in the chemical nature of the lipid matrix will influence the overall hydrophobicity which in turn influence particle size distribution. This is a crucial parameter for the nanoparticle formation that may have potential impact not only on the physical stability but also on drug loading capacity, drug release rate, rate of drug hydrolysis as well as in-vivo fate. Thus further basic research is in progress to study the effect of chemical nature lipid matrix on the physical stability of drug loaded SLN, chemical stability of the drug and its in-vivo fate.

\section{REFERENCES}

1- S. J. Lim and C. K. Kim, Int. J. Pharm., 243, 135-146 (2002).

2- M. Trotta, F. Debernardi and O. Caputo, ibid, 257, 153-160 (2003).

3- C. Schwarz, W. Mehnert, J. S. Lucks and R. H. Müller; J. Cont. Rel., 30, 83- 96 (1994).

4- Zetasizer 1000HS/3000HS Manual, Malvern Instruments Ltd., Malvern, Worcs. WR14 1XZ. United Kingdom.

5- M. A. Schubert and C. C. Müller-Goyman, Eur. J. Pharm. Biopharm., 55, 125-131 (2003).

6- T. Arnarson and P. H. Elworthy, J. Pharm. Pharmacology, 33, 141-144 (1981).

7- T. Arnarson and P. H. Elworthy, ibid, 32, 381-385 (1980).

8- K. M. Park, M. K. Lee, K. J. Hwang and C. K. Kim, Int. J. Pharm, 183, 145-154 (1999). 